\title{
Review
}

\section{Enhanced recovery after gynecologic surgery: review of the literature}

\author{
Mujde Can Ibanoglu ${ }^{1, *}$, Tugba Kinay ${ }^{1}$, Yaprak Engin-Ustun ${ }^{1}$ \\ ${ }^{1}$ Department of Obstetrics and Gynecology, Ankara Etlik Zubeyde Hanım Women's Health Training and Research Hospital, 06010 Ankara, Turkey \\ *Correspondence: drmujdecan@gmail.com (Mujde Can Ibanoglu) \\ Academic Editor: Donatella Caserta \\ Submitted: 26 March 2021 Revised: 30 May 2021 Accepted: 2 June 2021 Published: 11 February 2022
}

\begin{abstract}
Objective: Enhanced Recovery After Surgery (ERAS) includes practices aimed at providing rapid recovery by standardizing pre-and post-surgical care in patients undergoing colon surgery and reducing the metabolic stress response. The ERAS program is a systematic application that includes surgeons, anesthetists, nurses, and assistant healthcare professionals in the care process and has been successfully implemented in the international arena. Studies on ERAS protocols show that this system can be actively applied in different surgical areas. Mechanism: This review article discusses the main reviews and recommendations of the medical community on ERAS and valuable publications in the literature. Findings in brief: Guidelines of the ERAS Society for peri-operative care in gynecological procedures were first published in 2016 and updated in 2019. ERAS Society recommended 12 items for pre-and intra-operative care and 10 post-operative care items. The objectives of these applications are to provide patient education and consultancy services before surgery, to stop oral intake shortly before surgery and to start fast nutrition after surgery, to maintain a balanced body temperature and fluid level to maintain normal physiology, to increase mobilization, to provide pain control with painkillers, prophylaxis of nausea and vomiting and reduction of unnecessary or prolonged use of catheters. Conclusions: Studies suggest that increased compliance with ERAS agents in colon surgery has a positive effect on operation results, but more studies are needed to reach this affirmation in gynecological surgery.
\end{abstract}

Keywords: Enhanced recovery; Gynecology; Perioperative care; Surgery

\section{Introduction}

Gynecological surgery is very common; approximately 600,000 hysterectomies are performed each year in the USA for benign reasons [1]. The American College of Obstetricians and Gynecologists recommends surgery with the least invasive procedures to reduce cost and reduce complication rates [2]. Enhanced Recovery After Surgery guidelines has been adopted as peri-surgical care, bowel preparation, discontinuation of oral intake after midnight, use of narcotics, use of analgesia, and slow transition to oral intake with prolonged bed rest. This approach is not evidence-based and is thought to increase recovery time. For this reason, rapid surgical protocols are tried to be developed to maintain normal physiology in the perioperative period, reduce postoperative complications and improve patient outcomes without causing re-hospitalization.

Enhanced Recovery After Surgery (ERAS) includes practices aimed at providing rapid recovery by standardizing pre-and post-surgical care in patients undergoing colon surgery and reducing metabolic stress response [3]. The ERAS program is a systematic application that includes surgeons, anesthetists, nurses, and assistant healthcare professionals in the care process and has been successfully implemented in the international arena. Studies on ERAS protocols show that this system can be actively applied in different surgical areas [4].
ERAS in colorectal surgery enables a 2.5 -days reduction in hospitalization and a reduction of up to $50 \%$ in complications [5]. Using these protocols, an average profit of \$2245 (€1651) per patient was obtained [4,6]. Fast-track surgery protocols have been published on protocols applied for rectal, urological, pelvic, pancreatic, and stomach surgeries $[7,8]$.

Guidelines of the ERAS Society for peri-operative care in gynecological procedures were first published in 2016 and updated in 2019 [9]. ERAS Society recommended 12 items for pre-and intra-operative care and 10 post-operative care items. The objectives of these applications are to provide patient education and consultancy services before surgery, to stop oral intake shortly before surgery and to start fast nutrition after surgery, to maintain a balanced body temperature and fluid level to maintain normal physiology, to increase mobilization, to provide pain control with painkillers, prophylaxis of nausea and vomiting and reduction of unnecessary or prolonged use of catheters. Components of these guidelines are given in Fig. 1.

In this review, we briefly study the components and implementation of ERAS in benign gynecological surgery and discuss as a whole an improved perioperative outcome. 


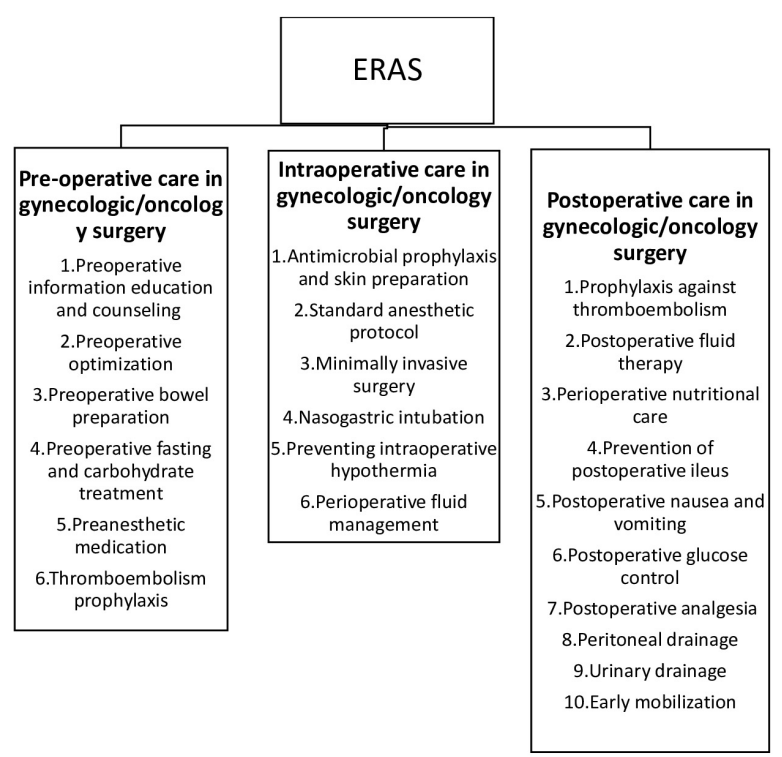

Fig. 1. Guidelines for perioperative care in gynecologic/oncology surgery: Enhanced Recovery After Surgery (ERAS ${ }^{\circledR)}$ Society recommendations.

\section{Pre-operative enhanced recovery after surgery components}

\subsection{Preoperative information education and counseling}

Patient education and counseling should include information to enlighten the patient about the operation and the recovery process. Components of effective patient education should be informed consent forms, including online models, and patient interviews with the surgical team. Although its benefit is not much in the studies, certainly, the consultancy service does not cause any harm; for this reason, pre-surgery patient education and counseling are recommended [10].

\subsection{Preoperative optimization}

Pre-operative health optimization includes medical comorbidities such as anemia and hyperglycemia. In addition, tobacco and alcohol use should be routinely evaluated before surgery. Population studies have shown that; $25 \%$ of women have anemia before elective hysterectomy or myomectomy [11-13]. Anemia is a factor associated with the high probability of blood transfusion during surgery and clinical adverse outcomes [12]. In a study on gynecological surgery, it was reported that anemia was associated with one-month mortality and morbidity [14]. Blood transfusion did not appear to improve these risks. Women with pre-operative hemoglobin $(\mathrm{Hgb})$ level $<12 \mathrm{~g} / \mathrm{dL}$ are more likely to be subjected to perioperative blood transfusions and their hospitalization time is also prolonged [15]. There are three goals in patient blood management, to keep the patient's pre-operative $\mathrm{Hgb}$ value of 12 and above, to reduce the amount of bleeding during surgery, and to be able to apply it correctly when blood transfusion is required [16]. Blood transfusion should be planned when the perioperative Hgb level falls below 7 [17]. Oral iron should be preferred over pre-operative blood transfusion [12]. There is evidence that interventions addressing these factors before elective surgery reduce perioperative morbidity and mortality [9].

Smoking and alcohol use are common lifestyle risk factors that can affect the outcome of the surgery, and they affect human physiology in different ways. Smoking mostly affects lung function, cardiovascular system, immune response, and tissue healing [18]. Alcohol negatively affects the liver, pancreas, and nervous system, heart function, immune capacity, hemostasis, metabolic stress response [19]. Smoking and alcohol are associated with increased postoperative morbidity and should be stopped at least four weeks before the procedure.

\subsection{Preoperative fasting and carbohydrate treatment}

Surgery is metabolic stress and therefore causes peripheral insulin resistance, resulting in hyperglycemia and postoperative complications, and prolonged recovery time [20]. In studies conducted in the light of this information, it has been shown that postoperative insulin resistance is reduced by half as a result of pre-operative carbohydrate loading sufficient for rapid insulin response [21]. Compared with fasting before the operation, it has been shown that patients who received carbohydrates were discharged earlier [21]. Oral carbohydrate loading reduces postoperative insulin resistance, improves preoperative health, and is routinely recommended [22].

\subsection{Preoperative bowel preparation}

ERAS protocols for gynecological surgery allow up to 2 hours of clear fluids and up to 6 hours of solids before induction of anesthesia, and carbohydrate loading is recommended [23]. Mechanical bowel preparation is not routinely recommended, even when bowel resection is planned; because mechanical bowel preparation can often cause dehydration and there is insufficient evidence that it will benefit the patient [24]. Studies have shown that the liquid taken up to 2 hours before surgery does not decrease the $\mathrm{pH}$ of gastric fluid, does not increase complications, and does not increase stomach content [25].

\subsection{Preanesthetic medication}

Routine sedatives should be avoided to reduce preoperative anxiety to speed up postoperative recovery [26]. Between the medications used in elective surgeries to be performed with general anesthesia and placebo, it has been shown that the postoperative experience of the patient does not improve and the duration of anesthesia is increased with these medications [27]. 


\subsection{Thromboembolism prophylaxis}

Patients at risk of venous thromboembolism (VTE) should receive prophylaxis with low-molecular-weight heparin (LMWH) or heparin initiated before surgery in combination with mechanical methods. Patients should discontinue oral contraception and switch to another form before surgery. Because in women using combined oral contraceptives thrombotic coagulation factor changes, it continues until 4-6 weeks after cessation of treatment [9, 26]. Therefore, it is recommended that the oral contraceptive method should be discontinued one month before the surgery, but this situation should be evaluated carefully with the possibility of unwanted pregnancy [26].

\section{Intra-operative enhanced recovery after surgery components}

\subsection{Antimicrobial prophylaxis and skin preparation}

Most pelvic surgeries include operations that belong to the clean-contaminated wound class. In gynecology, pathogens in surgical site infections often include skin flora, vaginal flora, or enteric bacteria. Intravenous antibiotics (1st generation cephalosporin or amoxicillinclavulanic acid) should be routinely administered within 60 minutes before skin incision [9]; additional doses should be given in case of prolonged operations, severe blood loss, and during the operation of obese patients [28]. For patients with penicillin/cephalosporin allergy, a combination of clindamycin and gentamicin or quinolone can be used [26]. Laparoscopic operations that are not contaminated with genitourinary or digestive tracts do not require antimicrobial prophylaxis. Before surgery, skin preparation is traditionally done with a shower, epilation, or skin antiseptic solution. However, there is no clear evidence that epilation reduces wound infection, regardless of the method chosen (shaving, haircutting, or depilatory cream) [28]. For this reason, epilation should be avoided and if necessary, a haircut is preferred. For skin cleansing, chlorhexidine-alcohol should be preferred over aqueous povidone-iodine solution [29].

\subsection{Standard anesthetic protocol}

Short-acting anesthetic agents are preferable during surgery to ensure rapid reanimation [9]. With the addition of regional anesthesia to general anesthesia, postoperative nausea and vomiting are reduced and a faster awakening is provided. Since tidal volume (TV) can cause ventilatorinduced lung damage, no systematic advice can be provided, therefore no comment will be made on this issue [8].

\subsection{Minimally invasive surgery}

Although minimally invasive surgery is an important factor in reducing hospital stay and accelerating patient recovery on its own, it is not seen as an integral part of ERAS. In the ERAS protocol, it is recommended that efforts be made to minimize surgical trauma by using the smallest incision possible, making a transverse incision instead of the sub-umbilical median [9].

\subsection{Nasogastric intubation}

While planning the operation within the scope of ERAS, the surgical team should pay attention to choosing the least invasive surgical method, determining the necessity and duration of bladder catheterization, avoiding nasogastric tubes, and minimizing the use of other drainage tubes $[8,30]$.

\subsection{Preventing intraoperative hypothermia}

Anesthesia models performed with the ERAS protocol also aim to maintain normothermia and perioperative euvolemia. Intraoperative hypothermia prolongs the recovery time after anesthesia. Maintaining normothermia can shorten the time in the post-anesthesia care unit and lower maintenance costs [31].

\subsection{Perioperative fluid management}

While the idea of replacing the losses in the body due to reduced urine output and unnoticeable sweating has traditionally been adopted, new studies have shown that these losses are not as high as thought [32]. Excess fluid therapy has also been shown to increase intravascular pressure and increase the atrial natriuretic peptide and cause deterioration in the vascular endothelium [33]. Consequently, the purpose of intraoperative fluid balance should be to maintain body weight before surgery, and fluid therapy with zero balance should be applied to ensure euvolemia [34].

\section{Post-operative enhanced recovery after surgery components}

Postoperative ERAS components generally focus on pain management, rapid recovery of bowel function, diet, and patient mobilization.

The primary expectation of patients who have undergone gynecological surgery due to benign indications is to be discharged within one to two days following the surgery. On the other hand, the second group recommendations of the ERAS group were weak compared to the first group recommendations in terms of the reliability of the data.

This has led to a weak evaluation of the evidence in studies including early mobilization and postoperative analgesia, especially in the selection of the optimal analgesic regimen in vaginal and open surgery, the necessity and duration of urinary drainage. In some cases, recommendations have been made based on findings from other surgical disciplines in which major abdominal surgery is routinely used. These comments should be taken into account when evaluating the Guidelines for postoperative care in gynecologic/oncology surgery: Enhanced Recovery After Surgery (ERAS $\left.{ }^{\circledR}\right)$ Society recommendations_-Part II mentioned below. 


\subsection{Early mobilization}

Patients should be encouraged to mobilize within 24 hours of surgery.

\subsection{Prophylaxis against thromboembolism}

Recommendations for postoperative venous thromboembolism prophylaxis are for patients to wear appropriate pressure stockings and apply intermittent pneumatic compression. Expanded prophylaxis for patients after laparotomy for abdominal or pelvic malignancies, that is, should be arranged as 28 days [35].

\subsection{Postoperative fluid therapy}

Intravenous fluids should be stopped within 24 hours after surgery and balanced crystalloid solutions should be preferred [35].

\subsection{Perioperative nutritional care}

A standard diet is recommended within the first 24 hours after surgery.

\subsection{Prevention of postoperative ileus and glucose control}

With the use of postoperative laxatives and chewing gum, rapid recovery of the intestines can be achieved [35]. In addition, by keeping blood glucose levels between 180$200 \mathrm{mg} / \mathrm{dL}$ before, during, and after the operation to reduce insulin resistance, metabolic stress can be reduced, and ultimately perioperative results can be improved [35].

\subsection{Postoperative analgesia, nausea, and vomiting}

A multimodal analgesia strategy should be applied to reduce the need for opioids after surgery and to cope with pain. Post-operatively, opioids should be given orally to patients as soon as possible [35]. Postoperative nausea and vomiting (PONV) is a common problem that delays recovery and prolongs hospital stay [36]. Women who have undergone laparoscopic and gynecological surgery, It has a high risk of PONV, especially in long-term operations [36]. As a pain reliever, acetaminophen and non-steroidal antiinflammatory drugs (NSAIDs) in combination or dexamethasone can be applied to all patients as long as there are no contraindications to reduce postoperative nausea and vomiting [35].

\subsection{Peritoneal and urinary drainage}

Urine catheters should be removed as soon as possible postoperatively and within a maximum of 24 hours. Patients should be encouraged to act within 24 hours after surgery [35].

Peritoneal drainage has traditionally been used to prevent fluid accumulation in the dissection bed, reduce blood, serous collections, or the rate of infection [35]. It has also been thought to be effective in preventing anastomotic leakage in colorectal surgery [37]. However, drainage is not routinely recommended after colon and rectal surgery
[38,39]. In the field of gynecology, there is very little data on drains and anastomotic leaks [40]. It is not very appropriate to comment on the data obtained from colorectal surgery in gynecological surgery [35]. In a Cochrane systematic review four studies with 571 participants were included [41]. Lymphocysts of drains after pelvic lymphadenectomy has been shown to inhibit [41]. Drains have even been associated with higher cyst rates after pelvic lymphadenectomy [41]. There are few studies on para-aortic lymphadenectomy, but no evidence to suggest drainage [42].

\section{Discussion}

ERAS guideline examines many peri and postoperative suggestions for different surgical branches. Studies have been conducted in which different protocols such as gynecological surgeries, vaginal procedures, minimally invasive surgery were performed. In addition, there are reviews to explain which elements are more important in these protocols applied to improve operative results. In all studies evaluating open benign hysterectomies, a reduction in hospital stay was observed with ERAS applications [43]. In the Wijk study, it was shown that the rate of discharge within 2 days increased in patients who underwent ERAS protocol and operated with open surgery [44]. In the next study of the same group, they showed that surgery performed for malignant and benign indications did not change the length of hospital stay [45]. In the study by Kondo et al. [46], when they compared the ERAS application in the intestinal infiltrating deep endometriosis group, it showed that it shortened the hospital stay. In conclusion, the length of stay for laparotomy, laparoscopy and vaginal surgery was shortened in the ERAS groups [46]. This resulted in an improvement in cost in all surgical groups. It also resulted in an increase in patient satisfaction and comfort [46]. When the groups with and without ERAS were compared, it was observed that there was a decrease in narcotic use in the ERAS group in all gynecological surgery groups [47].

In a study by Pache et al. [48], It was shown that there was at least a 70\% improvement in postoperative complications and hospital stay when acted in line with ERAS protocols, and it was also emphasized that these results were better in gynecological surgery compared to colorectal surgery.

In another study, gynecological operations were compared under the heading of regional anesthesia and general anesthesia applied in line with ERAS recommendations. The results showed that the duration of stay, complication rate, intravenous fluid use, and opioid use were reduced in the regional anesthesia group. Increased patient satisfaction and decreased hospital costs have been stated as additional benefits of ERAS [49].

In a recent report, each component related to the use of ERAS in gynecological surgery was examined separately. It was concluded that reduced hunger, skin preparation with chlorhexidine, multimodal pain management, nausea and 
Table 1. Review of the Literature.

\begin{tabular}{|c|c|c|}
\hline Author & Recommendations & Results \\
\hline Pache et al. [48] & All items. & $\begin{array}{l}70 \% \text { improvement in postoperative complications and } \\
\text { hospital stay when acted in line with ERAS protocols. }\end{array}$ \\
\hline Modesitt et al. [49] & Intraoperative anesthesia type. & $\begin{array}{l}\text { Duration of stay, complication rate, intravenous fluid use, } \\
\text { and opioid use was reduced in the regional anesthesia } \\
\text { group. }\end{array}$ \\
\hline Kalogera et al. [50] & $\begin{array}{l}\text { Patient education, bowel preparation, elimination of na- } \\
\text { sogastric tubes, minimization of surgical drains, early } \\
\text { postoperative mobilization, early postoperative feeding, } \\
\text { early intravenous fluid discontinuation, early removal of } \\
\text { urinary catheters, use of laxatives, chewing gum, sur- } \\
\text { gical site infection reduction bundle, glucose manage- } \\
\text { ment, and venous thromboembolism prophylaxis. }\end{array}$ & $\begin{array}{l}\text { Evidence and existing guidelines support } 29 \text { protocol ele- } \\
\text { ments for the Agency for Healthcare Research and Quality } \\
\text { Safety Program for Improving Surgical Care and Recov- } \\
\text { ery in gynecologic surgery. }\end{array}$ \\
\hline Ferrari et al. [8] & $\begin{array}{l}\text { ERAS protocols in malign and benign gynecological } \\
\text { surgeries. }\end{array}$ & $\begin{array}{l}\text { The advantages of ERAS in gynecologic surgery have } \\
\text { been strongly established, especially reduction in length } \\
\text { of stay. }\end{array}$ \\
\hline Scheib et al. [4] & $\begin{array}{l}\text { Preoperative counseling to the patient, no bowel prepa- } \\
\text { ration, an opioid-sparing multimodal approach to pain } \\
\text { management, goal-directed fluid management, mini- } \\
\text { mally invasive surgery when possible, and early mobi- } \\
\text { lization and feeding. }\end{array}$ & $\begin{array}{l}\text { This study supports quality evidence for individual com- } \\
\text { ponents of the ERAS pathway. }\end{array}$ \\
\hline Peters et al. [51] & $\begin{array}{l}\text { In laparoscopic minimally invasive gynecological pro- } \\
\text { cedures, the results of recovery with ERAS protocols } \\
\text { and traditional methods were compared. }\end{array}$ & $\begin{array}{l}\text { In this group of surgeries, there was no difference in } 30 \text { - } \\
\text { day morbidity between the two groups, but the discharge } \\
\text { rates increased on the same day as the surgery, and im- } \\
\text { provement in perioperative outcomes was shown. }\end{array}$ \\
\hline Bisch et al. [54] & $\begin{array}{l}\text { In this study, the results of the applications made ac- } \\
\text { cording to the recommendations in the guides related to } \\
\text { ERAS and the applications made before these guidelines } \\
\text { are compared. }\end{array}$ & $\begin{array}{l}\text { It has been observed that the clinical results have improved } \\
\text { and the money allocated for healthcare has been saved in } \\
\text { the applications carried out with the recommendations of } \\
\text { the ERAS guidelines. }\end{array}$ \\
\hline Wijk et al. [56] & $\begin{array}{l}\text { This study is comparing postoperative results with En- } \\
\text { hanced Recovery After Surgery Gynecologic/Oncology } \\
\text { guideline. }\end{array}$ & $\begin{array}{l}\text { Improvement in clinical outcomes has been shown with } \\
\text { compliance with the Enhanced Recovery After Surgery } \\
\text { Gynecologic/Oncology guideline elements. }\end{array}$ \\
\hline
\end{tabular}

vomiting prophylaxis, antibiotic prophylaxis, fluid balance management, maintenance of body temperature at normal values, reduction of drain use and transition to early nutrition are feasible with a high level of evidence. Applications with moderate evidence value were listed as carbohydrate loading, avoidance of bowel preparation, use of prokinetics, and early mobilization [50].

In another study evaluating fifty studies examining ERAS in gynecological surgery; It was stated that it is important to provide the patient with preoperative consultancy, not to make bowel preparation, multimodal approach in pain management, fluid balance management, preferring minimally invasive surgery as much as possible, and early mobilization and nutrition. In this study, multidisciplinary teamwork and active participation of the patient in the process are also emphasized [4].

In a study in which 410 women who had undergone gynecological surgery were examined, it was reported that the same-day discharge rate increased by $9.4 \%$ with ERAS applications. It has been reported that pre-operative antiemetic applications and applications during anesthesia provide a decrease in postoperative pain and nausea/vomiting $(p<0.001)$. It was stated that the increase in discharge rates on the same day did not cause any change in postoperative complications or re-hospitalization rates [51].

COVID-19 has a high and increasing attack rate and was announced as a pandemic by World Health Organization (WHO). The symptoms of the disease caused by the responsible virus, SARS-CoV-2 exhibit a wide spectrum of manifestations from asymptomatic patients to severe pneumonitis with multiple organ failure. Depending on the disease burden of the society, the rate of asymptomatic carriers among adults admitted for surgery varies between $0.5 \%$ and $2 \%$ [52]. It is imperative to identify patients who are asymptomatic but infected with SARS-CoV-2. The operations of such patients can be delayed safely. Thus, patients, health- 
care workers, and other patients can be protected by avoiding unnecessary exposures. Bedside preoperative COVID19 screening should be integrated into the ERAS program so that we can reduce the risk of horizontal viral transmission and reduce the perioperative complications from unrecognized SARS-CoV-2 infection [53].

ERAS Society's guidelines for peri-operative care in gynecological surgery [54], published in 2016, provide recommendations for pre-and intra-operative care and postoperative care, and the recommendations in these two guidelines are based on colorectal surgery guidelines. Studies suggest that increased compliance with ERAS agents in colon surgery has a positive effect on operation results, but more studies are needed to reach this affirmation in gynecological surgery [55]. Table 1 (Ref. [4,8,48-51,54,56]) provides a summary of the studies on ERAS.

As stated by the association, ERAS is multidisciplinary management of an unstable and dynamic process that needs to be improved as the results emerge. When the literature is reviewed, there is still a need for large randomized studies in which different surgical techniques are compared and the elements of the protocol are discussed one by one. It is not clear which items are more critical in the ERAS program [57]. Few studies have evaluated the results of ERAS protocols in demographics such as obese, thin, old, or young. Accordingly, it is necessary to develop evidence-based components for perioperative care with ERAS recommendations, and it is necessary to develop studies that will serve this purpose.

\section{Author contributions}

TK designed the study. MCI performed the research and data collection. YEU provided help and advice on the analyzed the data. All of the authors, MCI, TK, YEU have contributed to writing of the manuscript. read and approved the final manuscript.

\section{Ethics approval and consent to participate}

Not applicable.

\section{Acknowledgment}

Thanks to all the peer reviewers for their opinions and suggestions.

\section{Funding}

This research received no external funding.

\section{Conflict of interest}

The authors declare no conflict of interest.

\section{References}

[1] Wu JM, Wechter ME, Geller EJ, Nguyen TV, Visco AG. Hysterectomy rates in the United States, 2003. Obstetrics and Gynecology. 2007; 110: 1091-1095.
[2] Dayaratna S, Goldberg J, Harrington C, Leiby BE, McNeil JM Hospital costs of total vaginal hysterectomy compared with other minimally invasive hysterectomy. American Journal of Obstetrics and Gynecology. 2014; 210: 120.e1-120.e6.

[3] Kehlet H, Wilmore DW. Evidence-Based Surgical Care and the Evolution of Fast-Track Surgery. Annals of Surgery. 2008; 248: 189-198.

[4] Scheib SA, Thomassee M, Kenner JL. Enhanced Recovery after Surgery in Gynecology: a Review of the Literature. Journal of Minimally Invasive Gynecology. 2019; 26: 327-343.

[5] Ljungqvist O. Jonathan E. Rhoads lecture 2011: Insulin resistance and enhanced recovery after surgery. Journal of Parenteral and Enteral Nutrition. 2012; 36: 389-398.

[6] Adamina M, Kehlet H, Tomlinson GA, Senagore AJ, Delaney CP. Enhanced recovery pathways optimize health outcomes and resource utilization: a meta-analysis of randomized controlled trials in colorectal surgery. Surgery. 2011; 149: 830-840.

[7] Nygren J, Thacker J, Carli F, Fearon KCH, Norderval S, Lobo $\mathrm{DN}$, et al. Guidelines for perioperative care in elective rectal/pelvic surgery: Enhanced Recovery after Surgery (ERAS $\left.{ }^{\circledR}\right)$ Society recommendations. Clinical Nutrition. 2012; 31: 801816.

[8] Ferrari F, Forte S, Sbalzer N, Zizioli V, Mauri M, Maggi C, et al. Validation of an enhanced recovery after surgery protocol in gynecologic surgery: an Italian randomized study. American Journal of Obstetrics and Gynecology. 2020; 223: 543.e1-543.e14.

[9] Nelson G, Altman AD, Nick A, Meyer LA, Ramirez PT, Achtari $\mathrm{C}$, et al. Guidelines for pre- and intra-operative care in gynecologic/oncology surgery: Enhanced Recovery After Surgery (ERAS $\left.{ }^{\circledR}\right)$ Society recommendations-Part I. Gynecologic Oncology. 2015; 140: 313-22.

[10] van der Meij E, Anema JR, Leclercq WKG, Bongers MY, Consten ECJ, Schraffordt Koops SE, et al. Personalised perioperative care by e-health after intermediate-grade abdominal surgery: a multicentre, single-blind, randomised, placebocontrolled trial. Lancet. 2018; 392: 51-59.

[11] Muñoz M, Laso-Morales MJ, Gómez-Ramírez S, Cadellas M, Núñez-Matas MJ, García-Erce JA. Pre-operative haemoglobin levels and iron status in a large multicentre cohort of patients undergoing major elective surgery. Anaesthesia. 2017; 72: 826834.

[12] Stone R, Carey E, Fader AN, Fitzgerald J, Hammons L, Nensi A, et al. Enhanced Recovery and Surgical Optimization Protocol for Minimally Invasive Gynecologic Surgery: an AAGL White Paper. Journal of Minimally Invasive Gynecology. 2021; 28: 179-203.

[13] Murji A, Lam M, Allen B, Richard L, Shariff SZ, Austin PC, et $a l$. Risks of preoperative anemia in women undergoing elective hysterectomy and myomectomy. American Journal of Obstetrics and Gynecology. 2019; 221: 629.e1-629.e18.

[14] Richards T, Musallam KM, Nassif J, Ghazeeri G, Seoud M, Gurusamy KS, et al. Impact of Preoperative Anaemia and Blood Transfusion on Postoperative Outcomes in Gynaecological Surgery. PLoS ONE. 2015; 10: e0130861.

[15] Clevenger B, Mallett SV, Klein AA, Richards T. Patient blood management to reduce surgical risk. the British Journal of Surgery. 2015; 102: 1325-1337.

[16] Partridge J, Harari D, Gossage J, Dhesi J. Anaemia in the older surgical patient: a review of prevalence, causes, implications and management. Journal of the Royal Society of Medicine. 2013; 106: 269-277.

[17] World Health Organization. Haemoglobin concentrations for the diagnosis of anaemia and assessment of severity. World Health Organization. 2011. Available at: http://www.who.int/vmnis/in dicators/haemoglobin/en/index.html (Accessed: 1 March 2013).

[18] Sorensen LT, Karlsmark T, Gottrup F. Abstinence from Smoking 
Reduces Incisional Wound Infection. Annals of Surgery. 2003; 238: $1-5$.

[19] Tønnesen H. Alcohol abuse and postoperative morbidity. Dan Med Bull. 2003; 50: 139-160.

[20] Nygren J. The metabolic effects of fasting and surgery. Best Practice \& Research. Clinical Anaesthesiology. 2006; 20: 429438

[21] Ljungqvist $\mathrm{O}$, Nygren $\mathrm{J}$, Thorell A. Modulation of postoperative insulin resistance by pre-operative carbohydrate loading. Proceedings of the Nutrition Society. 2002; 61: 329-336.

[22] Smith MD, McCall J, Plank L, Herbison GP, Soop M, Nygren J. Preoperative carbohydrate treatment for enhancing recovery after elective surgery. Cochrane Database of Systematic Reviews. 2014; CD009161.

[23] American College of Chest Physicians/Society of Critical Care Medicine Consensus Conference: definitions for sepsis and organ failure and guidelines for the use of innovative therapies in sepsis. Critical Care Medicine. 1992; 20: 864-874.

[24] Bretagnol F, Panis Y, Rullier E, Rouanet P, Berdah S, Dousset B, et al. Rectal Cancer Surgery with or without Bowel Preparation. Annals of Surgery. 2010; 252: 863-868.

[25] Smith I, Kranke P, Murat I, Smith A, O'Sullivan G, Sreide E, et al. Perioperative fasting in adults and children. European Journal of Anaesthesiology. 2011; 28: 556-569.

[26] ACOG Committee Opinion No. 750: Perioperative Pathways: Enhanced Recovery After Surgery. Obstetrics \& Gynecology. 2018; 132: e120-e130.

[27] Maurice-Szamburski A, Auquier P, Viarre-Oreal V, Cuvillon P, Carles M, Ripart J, et al. Effect of sedative premedication on patient experience after general anesthesia: a randomized clinical trial. The Journal of the American Medical Association. 2015; 313: 916-925.

[28] ACOG Practice Bulletin No. 195: Prevention of Infection After Gynecologic Procedures. Obstetrics \& Gynecology. 2018; 131: e172-e189.

[29] Facts D. Comparisons. St. Louis, MO, USA: Wolters kluwer health, inc. 2005. Available at: www.factsardcomparisons.com (Accessed: 20 May 2013).

[30] Bauer VP. The Evidence against Prophylactic Nasogastric Intubation and Oral Restriction. Clinics in Colon and Rectal Surgery. 2013; 26: 182-185.

[31] Kurz A, Sessler DI, Narzt E, Bekar A, Lenhardt R, Huemer G, et al. Postoperative hemodynamic and thermoregulatory consequences of intraoperative core hypothermia. Journal of Clinical Anesthesia. 1995; 7: 359-366.

[32] SHIRES T, WILLIAMS J, BROWN F. Acute Change in Extracellular Fluids Associated with Major Surgical Procedures. Annals of Surgery. 1961; 154: 803-810.

[33] Becker BF, Chappell D, Jacob M. Endothelial glycocalyx and coronary vascular permeability: the fringe benefit. Basic Research in Cardiology. 2010; 105: 687-701.

[34] Brandstrup B, Svendsen PE, Rasmussen M, Belhage B, Rodt $\mathrm{S} \AA$, Hansen B, et al. Which goal for fluid therapy during colorectal surgery is followed by the best outcome: near-maximal stroke volume or zero fluid balance? British Journal of Anaesthesia. 2012; 109: 191-199.

[35] Nelson G, Altman AD, Nick A, Meyer LA, Ramirez PT, Achtari $\mathrm{C}$, et al. Guidelines for postoperative care in gynecologic/oncology surgery: Enhanced Recovery after Surgery (ERAS ${ }^{\circledR)}$ ) Society recommendations-Part II. Gynecologic Oncology. 2016; 140: 323-332.

[36] Chiu C, Aleshi P, Esserman LJ, Inglis-Arkell C, Yap E, Whitlock EL, et al. Improved analgesia and reduced post-operative nausea and vomiting after implementation of an enhanced recovery after surgery (ERAS) pathway for total mastectomy. BMC Anesthesiology. 2018; 18: 41.
[37] Karliczek A, Jesus EC, Matos D, Castro AA, Atallah AN, Wiggers T. Drainage or nondrainage in elective colorectal anastomosis: a systematic review and meta-analysis. Colorectal Disease. 2006; 8: 259-265.

[38] Jesus EC, Karliczek A, Matos D, Castro AA, Atallah AN. Prophylactic anastomotic drainage for colorectal surgery. The Cochrane Database of Systematic Reviews. 2004; CD002100.

[39] Petrowsky H, Demartines N, Rousson V, Clavien P. Evidencebased Value of Prophylactic Drainage in Gastrointestinal Surgery. Annals of Surgery. 2004; 240: 1074-1085.

[40] Kalogera E, Dowdy SC, Mariani A, Aletti G, Bakkum-Gamez JN, Cliby WA. Utility of closed suction pelvic drains at time of large bowel resection for ovarian cancer. Gynecologic Oncology. 2012; 126: 391-396.

[41] Charoenkwan K, Kietpeerakool C. Retroperitoneal drainage versus no drainage after pelvic lymphadenectomy for the prevention of lymphocyst formation in women with gynaecological malignancies. The Cochrane Database of Systematic Reviews. 2017; 6: CD007387.

[42] Morice P, Lassau N, Pautier P, Haie-Meder C, Lhomme C, Castaigne D. Retroperitoneal drainage after complete Para-aortic lymphadenectomy for gynecologic cancer: a randomized trial. Obstetrics and Gynecology. 2001; 97: 243-247.

[43] Mukhopadhyay D. Enhanced recovery programme in gynaecology: outcomes of a hysterectomy care pathway. BMJ Quality Improvement Reports. 2015; 4.

[44] Wijk L, Franzen K, Ljungqvist O, Nilsson K. Implementing a structured Enhanced Recovery after Surgery (ERAS) protocol reduces length of stay after abdominal hysterectomy. Acta Obstetricia et Gynecologica Scandinavica. 2014; 93: 749-756.

[45] Wijk L, Franzén K, Ljungqvist O, Nilsson K. Enhanced Recovery after Surgery Protocol in Abdominal Hysterectomies for Malignant versus Benign Disease. Gynecologic and Obstetric Investigation. 2016; 81: 461-467.

[46] Kondo W, Ribeiro R, Zomer MT. Fast-track surgery in intestinal deep infiltrating endometriosis. Journal of Minimally Invasive Gynecology. 2014; 21: 285-290.

[47] Brummett CM, Waljee JF, Goesling J, Moser S, Lin P, Englesbe MJ, et al. New Persistent Opioid Use after Minor and Major Surgical Procedures in us Adults. JAMA Surgery. 2017; 152: e170504.

[48] Pache B, Jurt J, Grass F, Hübner M, Demartines N, Mathevet P, et al. Compliance with enhanced recovery after surgery program in gynecology: are all items of equal importance? International Journal of Gynecological Cancer. 2019; 29.

[49] Modesitt SC, Sarosiek BM, Trowbridge ER, Redick DL, Shah PM, Thiele RH, et al. Enhanced Recovery Implementation in Major Gynecologic Surgeries. Obstetrics \& Gynecology. 2016; 128: 457-466.

[50] Kalogera E, Nelson G, Liu J, Hu QL, Ko CY, Wick E, et al. Surgical technical evidence review for gynecologic surgery conducted for the Agency for Healthcare Research and Quality Safety Program for Improving Surgical Care and Recovery. American Journal of Obstetrics and Gynecology. 2018; 219: 563.e1-563.e19.

[51] Peters A, Siripong N, Wang L, Donnellan NM. Enhanced recovery after surgery outcomes in minimally invasive nonhysterectomy gynecologic procedures. American Journal of Obstetrics and Gynecology. 2020; 223: 234.e1-234.e8.

[52] Morris M, Pierce A, Carlisle B, Vining B, Dobyns J. Preoperative COVID-19 testing and decolonization. The American Journal of Surgery. 2020; 220: 558-560.

[53] Stone R, Scheib S. Advantages of, and Adaptations to, Enhanced Recovery Protocols for Perioperative Care during the COVID19 Pandemic. Journal of Minimally Invasive Gynecology. 2020; 28: 481-489. 
[54] Bisch SP, Wells T, Gramlich L, Faris P, Wang X, Tran DT, et al. Enhanced Recovery after Surgery (ERAS) in gynecologic oncology: System-wide implementation and audit leads to improved value and patient outcomes. Gynecologic Oncology. 2018; 151: $117-123$.

[55] Kalogera E, Dowdy SC. Enhanced Recovery Pathway in Gynecologic Surgery: Improving Outcomes through Evidence-Based Medicine. Obstetrics and Gynecology Clinics of North America. 2016; 43: 551-573.
[56] Nelson G, Dowdy SC, Lasala J, Mena G, Bakkum-Gamez J, Meyer LA, et al. Enhanced recovery after surgery (ERAS ${ }^{\circledR}$ ) in gynecologic oncology - Practical considerations for program development. Gynecologic Oncology. 2017; 147: 617-620.

[57] Wijk L, Udumyan R, Pache B, Altman AD, Williams LL, Elias $\mathrm{KM}$, et al. International validation of Enhanced Recovery After Surgery Society guidelines on enhanced recovery for gynecologic surgery. American journal of obstetrics and gynecology. 2019; 221: 237. e1-237. e11. 